\title{
Microscopic and Chemical Assessments of the Filling Ability in Oval-Shaped Root Canals Using Two Different Carrier-Based Filling Techniques
}

\author{
Davide Mancino ${ }^{1,2}$ Naji Kharouf ${ }^{1,2}$ Joseph Hemmerlé ${ }^{1,2} \quad$ Youssef Haïkel $^{1,2}$ \\ ${ }^{1}$ Department of Endodontics, Faculty of Dental Medicine, \\ Strasbourg University, Strasbourg, France \\ 2UMR-S 1121 Inserm, Biomaterials and Bioengineering, Strasbourg, \\ Address for correspondence Davide Mancino, DDS, PhD, \\ 1 rue place de l'Hôpital, Departement of Endodontics, \\ faculty of Dental Medicine, 67000 Strasbourg, France \\ (e-mail: endodontiefrancaise@outlook.com).
}

Eur J Dent 2019;13:166-171

\begin{abstract}
Objectives The aim of this study was to assess the filling ability in oval-shaped canals using two different carrier-based filling techniques.

Materials and Methods Twenty-four human mandibular premolars with one oval canal were selected. Canals were shaped using WaveOne Gold Primary and ProGlider. Samples were divided into two groups and filled as follows: Thermafil and GuttaCore. The proportions of gutta-percha-filled areas (GPFAs), sealer-filled areas (SFAs), and void areas (VA), at 2 and $5 \mathrm{~mm}$, were analyzed using optical numeric microscope, scanning electron microscope, and energy-dispersive $\mathrm{X}$-ray.

Statistical Analysis Data were compared by Kruskal-Wallis one-way analysis of variance on ranks, with statistical significance set at $\alpha=0.05$.

Results At 2 and $5 \mathrm{~mm}$ distances from the apex, this study discloses no statistically different filling ability for the two techniques. Concerning each sample treated using both filling systems, the presence of tags was visualized. At working length (WL)- 5 , and WL-2, the maximum tag penetration depth for the GuttaCore group into the den-

Keywords

- carrier-based techniques

- filling ability

- GuttaCore

- oval-shaped canals

- Thermafil tinal tubules was, respectively, $96 \mu \mathrm{m}$ and $48 \mu \mathrm{m}$, whereas the values in the thermafil group were $109 \mu \mathrm{m}$ and $55 \mu \mathrm{m}$, respectively.

Conclusions Our results clearly show that Thermafil and GuttaCore can fill ovalshaped canals in appropriate way. Furthermore, we can state that the GuttaCore obturator allows to preserve the same filling ability than Thermafil obturator, in view of the fact that there was no difference, in terms of GPFA, SFA, and VA between the two different carrier-based obturation techniques.
\end{abstract}

\section{Introduction}

A successful root canal treatment depends on appropriate access cavity preparation, suitable shaping, proper cleaning, and tridimensional filling of the root canal system. ${ }^{1-9}$ Although literature highlights the importance of shaping and cleaning step during the endodontic therapy, there is no evidence other than clinical feeling to support or deny any particular filling technique. ${ }^{10-14}$ Thus, the role played by the filling step is inevitably underestimated. Often the choice of a filling technique is based on factors such as speed, simplicity, or economics, rather than on the endodontic anatomy or on other quantitative parameters. ${ }^{15}$ Without the slightest doubt, a tridimensional shaping and cleaning of the root canal system are essential requirements to perform a faultless filling. ${ }^{13,15}$

The root canal filling should be regarded as the final step of the disinfection. No cleaning technique allows to eradicate $100 \%$ of bacteria present in an infected root canal system. ${ }^{16-23}$ 
The task of canal filling must allow to entomb the remaining bacteria, to ensure the periapical healing as long as possible. ${ }^{24}$ To provide a tridimensional seal, the root canal filling involves the use of gutta-percha in combination with a root canal sealer. The sealer is used to fill the voids and gaps remaining between the gutta-percha material and the root canal walls, like a gasket, especially in oval- or irregular-shaped root canals. On the other hand, the sealer represents the weak link in the chain of root canal filling for three main reasons: first of all, for its setting reaction with subsequent shrinkage, then for its intrinsic porosities and at last for its possible reabsorption over time. ${ }^{15,19}$ These factors are likely the cause of void areas' (VA) formation that, of course, constitute a relevant problem in terms of sealing. Hence, the sealer thickness should be as smaller as possible. In this respect warm gutta-percha obturation techniques, and in particular Thermafil system (Dentsply Sirona), have been developed to perform tridimensional root canal filling with a smaller sealer thickness. ${ }^{11,12}$ However, the presence of its plastic carrier could be a real problem. First, the clinicians, who lack of the basic principles for use Thermafil obturator, could perform a root canal filling with a naked plastic carrier in the last millimeter of the canal. ${ }^{25,26}$ Second, the preparation of the postspace, if necessary, in a canal filled with a Thermafil obturator could be very difficult and could cause a postoperative perforation as result of a lack of attention or experience on the part of the clinician. Third, the retreatment management in a tooth filled with a Thermafil obturator could be very difficult for a general practitioner. For these reasons, we decided to test two different techniques of carrier-based obturation: thermafil and GuttaCore (Dentsply Sirona). GuttaCore is a carrier-based obturator and it has emerged as an effective filling system.

\section{Materials and Methods}

This study was revised and approved by the Ethics Committee of Medical, Odontology school, and Strasbourg University Hospital (protocol no. 2018-89).

A total of 87 mandibular first premolars, freshly extracted for orthodontic reasons, with a total length between 20 and $22 \mathrm{~mm}$, were selected. Informed consent was obtained from all patients, and all the procedures were performed according to the Helsinki Declaration of 1975. After debridement of the root surface, specimens were immersed in a $1 \%$ sodium hypochlorite $(\mathrm{NaOCl})$ solution at $4^{\circ} \mathrm{C}$ for 24 hours and then stored in saline solution.

Periapical radiographs were taken, placing the premolars according to their buccolingual and mesiodistal diameter to preselect the teeth with a long/short diameter ratio $\geq 1.5$ at $5 \mathrm{~mm}$ from the apex. Preselected teeth were then scanned with cone-beam computed tomography (CBCT) to select the teeth with the following morphological parameters:

- Presence of one canal

- A real oval canal at $5 \mathrm{~mm}$ from the apex with a long/ short diameter ratio $\geq 1.5$ at $5 \mathrm{~mm}$ from the apex

- Canal with a total length of $10 \pm 2 \mathrm{~mm}$ from canal orifice to apical foramen
- $10^{\circ}$ to $30^{\circ}$ primary root curvature in buccolingual and mesiodistal view according to the Schneider method $^{3}$

- Main curvature radius $\geq 4 \mathrm{~mm}^{4}$

- Therefore, only 28 mandibular first premolars ensured inclusion criteria, 4 were discarded because of severe calcification of the root canal, leaving a total of 24 teeth

- The tooth length was standardized to $18 \mathrm{~mm}$ by removing the coronal portion of the teeth

- Teeth having canals with apical diameters larger than size 20 were discarded.

\section{Shaping Procedure}

Specimens were mounted on Protrain (Simit Dental srl, Mantova, Italia) to perform endodontic therapy simulating clinical situation.

After access cavity preparation, all root canals were shaped, keeping the pulp chamber constantly flooded with $6 \%$ sodium hypochlorite. An experienced endodontist shaped each premolar. Canal scoutings were performed with a \#10 $\mathrm{K}$ file at a working length $(\mathrm{WL})+0.5 \mathrm{~mm}$. Length determination was obtained using an electronic apex locator (Root ZX; J Morita Co, Kyoto, Japan). Then, the apex was dried and sealed with a cyanoacrylate glue to simulate a closed system.

Mechanical glide path was performed using ProGlider (Dentsply Sirona) and an endodontic engine (X-Smart-IQ motor, Dentsply Sirona) at $300 \mathrm{rpm}$ and $4 \mathrm{Ncm}$ at the full WL.

Each tooth was shaped at the WL, using WaveOne Gold Primary (Dentsply Sirona) (0.25 tip, 0.07 taper) and an X-Smart-IQ motor (300 rpm, 4 Ncm).

New instruments were used to shape each premolar, and instruments were removed from the canal and cleaned after every two pecking motions until the WL was reached.

Recapitulation was performed between each instrument, taking a \#10 K file to WL $+0.5 \mathrm{~mm}$.

After the shaping procedure, to assure a tridimensional cleaning of the root canal system, an aqueous $17 \%$ solution of ethylenediaminetetraacetic acid was flooded into the pulp chamber and was then activated using a dynamic activation by EndoActivator (Dentsply Sirona) for 120 seconds in each canal. After rinsing with physiological saline, a $6 \%$ solution of $\mathrm{NaOCl}$ flooded into the pulp chamber was activated using EndoActivator for 120 seconds in each canal. Again, each canal was rinsed using physiological saline and then dried using sterile paper points.

The prepared teeth were randomly divided in two equal groups ( $n=12$ per group): thermafil group (TG) and GuttaCore group (GG).

\section{Thermafil System Group}

After application of a standardized drop (0.230 $\pm 0.005 \mathrm{~g})$ of AH Plus sealer (Dentsply Sirona) with a coated sterile paper point at the entrance of each canal, a second sterile paper point, taken to $\mathrm{WL}$, was used to remove any excess of sealer and smoothly distribute the sealer in the whole canal system. We placed the silicone stopper at the established WL on the primary WaveOne Gold Thermafil.

A Thermafil obturator (Dentsply Sirona) was heated in a ThermaPrep Plus oven until the first "beep" signal, indicating 
it is ready for use. The obturator was then directly inserted into the canal orifice with gentle apical pressure, without twisting or forcing, to the WL.

\section{GuttaCore Pink Obturator Group}

After application of a standardized drop (0.230 $\pm 0.005 \mathrm{~g})$ of AH Plus sealer (Dentsply Sirona) with a coated sterile paper point at the entrance of each canal, a second sterile paper point, taken to WL, was used to remove any excess of sealer and smoothly distribute the sealer in the whole canal system. With the help of the calibration rings on the WaveOne obturator, we placed the silicone stopper at the established WL.

A WaveOne Gold obturator (Dentsply Sirona) was heated in a GuttaCore Oven (Dentsply Sirona) until the first "beep" signal, indicating it is ready for use. The obturator was then directly inserted into the canal orifice with gentle apical pressure, without twisting or forcing, to the WL.

\section{Sectioning and Surface Measurements}

After embedding in Epon 812 resin (Euromedex; Souffelweyersheim, France), two sections were made perpendicular to the longitudinal axis of the root tooth at 2 and $5 \mathrm{~mm}$ from the apex with a wire saw (Walter EBNER; Le Locle, Switzerland). Thereafter, 1200, 2400, and 4000 P-grade (number of abrasive grains per $\mathrm{cm}^{2}$ ) abrasive papers were used successively to polish the surface. All samples were etched with a $20 \%(\mathrm{w} / \mathrm{w})$ citric acid solution for 2 minutes (Sigma-Aldrich, Darmstadt, Germany), thoroughly rinsed with distilled water, and finally dehydrated in a graded series of ethanol solutions.

\section{Optical Numeric Microscope Analysis}

Samples were first observed using an optical numeric microscope (KEYENCE; Osaka, Japan) and then analyzed using the VHX-5000 Communication software (KEYENCE; Osaka, Japan) to measure the quantity of voids and sealer filled areas.

\section{Scanning Electron Microscope Analysis}

Specimens were prepared for scanning electron microscopy (SEM) and sputter-coated with a gold-palladium alloy (20/80 weight\%) by using a HUMMER JR sputtering device (Technics; California, United States). The coated samples were observed with a Quanta 250 FEG SEM (FEI Company, Eindhoven, the Netherlands) operating with an accelerating voltage of the electrons of $10 \mathrm{kV}$.

\section{Energy Dispersive X-Ray Analysis}

The specimens also underwent chemical analysis to analyze the GuttaCore material and the related tags. Energy dispersive X-ray (EDX) microanalysis was performed with an EDAX Octane Plus spectrometer (AMETEK Materials Analysis Division, 91 McKee Dr, Mahwah, New Jersey, United States).

\section{Statistical Analysis}

Collected data were analyzed with SigmaPlot release 11.2 (Systat Software, Inc., San Jose, California, United States). The proportions of voids and sealer areas corresponding to one of two treatments GuttaCore (GC) or Thermafill (TH) were compared by Kruskal-Wallis one-way analysis of variance on ranks, with statistical significance set at $\alpha=0.05$.

\section{Results}

\section{Optical Microscope Observations}

For both groups, the superficies of gutta-percha-filled areas (GPFAs), sealer-filled areas (SFAs), and voids, that is, unfilled areas (VA) in the canal at WL-5 and WL-2 were observed and measured. All measurements are expressed in mean \pm standard error on the mean $(n=12)$.

At WL-5, concerning TG group, the VA was $0.22 \pm 0.001 \%$, the SAF was $0.74 \pm 0.003 \%$, and the GPFA was $99.04 \pm 0.02 \%$. Concerning the GG, the VA was $0.20 \pm 0.001 \%$, the SAF was $0.61 \pm 0.003 \%$, and the GPFA was $99.19 \pm 0.003 \%$.

At WL-2, concerning TG group, the VA was $0.13 \pm 0.004 \%$, the SAF was $1.54 \pm 0.01 \%$, and the GPFA was $98.33 \pm 0.04 \%$. Concerning the GG, the VA was $0.15 \pm 0.001 \%$, the SAF was $1.09 \pm 0.004 \%$, and the GPFA was $98.76 \pm 0.004 \%$.

\section{Scanning Electron Microscope Results}

SEM analyses ( - Figs. 1 and $\mathbf{2}$ ) confirmed the results obtained by optical microscopy. Furthermore, concerning each sample treated using both filling systems, the presence of tags was visualized (-Figs. 3 and 4). At WL-5 and WL-2, the maximum tag penetration depth for the GuttaCore into the

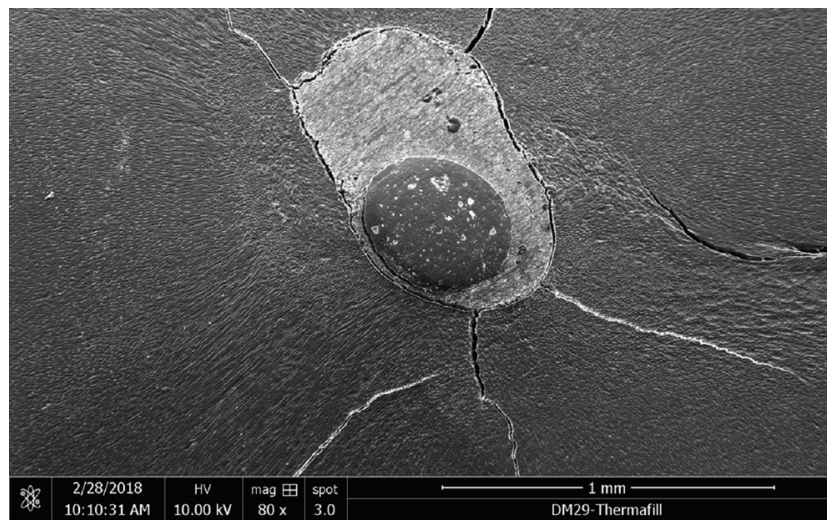

Fig. 1 Scanning electron micrograph of sectioned root at $5 \mathrm{~mm}$ from the apex filled with Thermafil obturator.

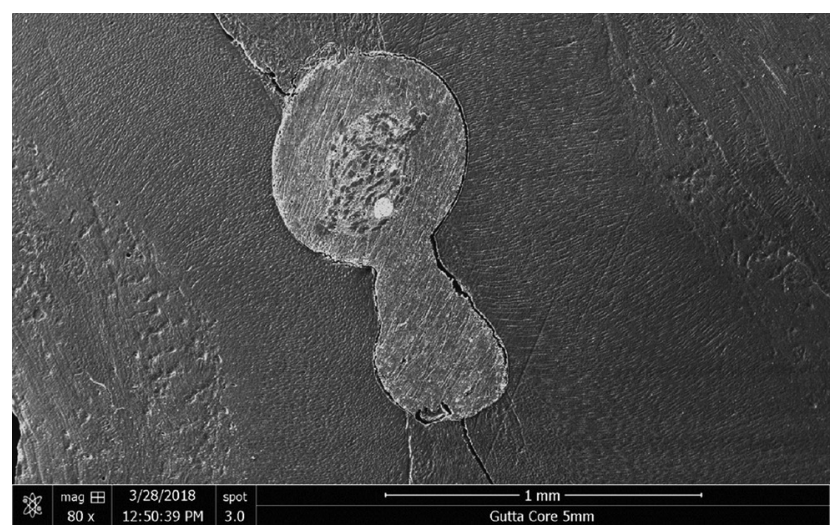

Fig. 2 Scanning electron micrograph of sectioned root at $5 \mathrm{~mm}$ from the apex filled with GuttaCore obturator. 


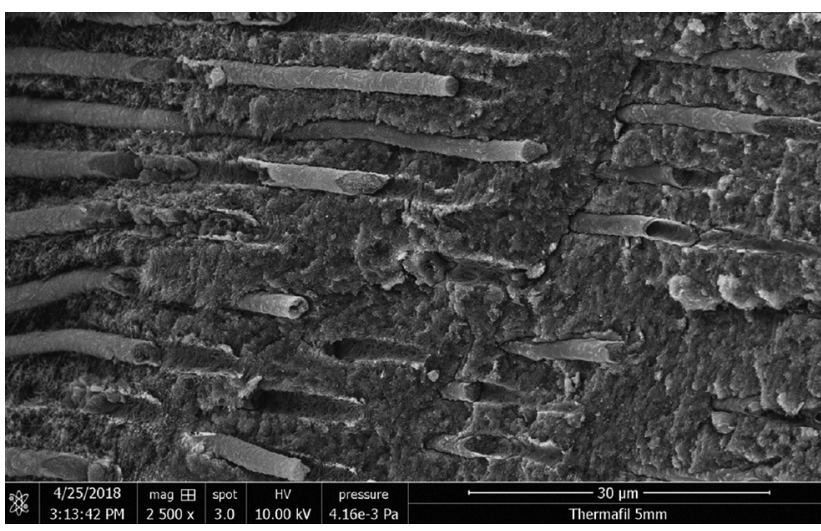

Fig. 3 Scanning electron micrograph showing the penetration of the gutta-percha in the dentinal tubules on extracted tooth filled with a Thermafil obturator.

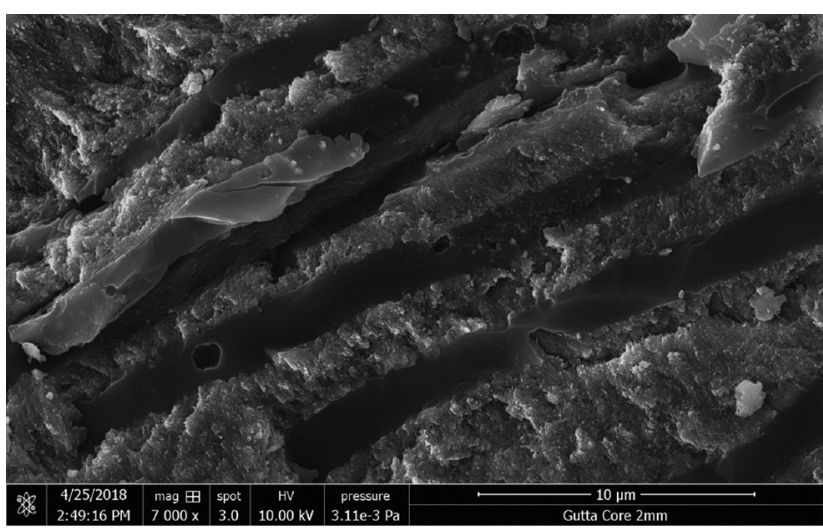

Fig. 4 Scanning electron micrograph showing the penetration of the gutta-percha in the dentinal tubules on extracted tooth filled with a GuttaCore obturator.

dentinal tubules was, respectively, 96 and $48 \mu \mathrm{m}$, whereas for the Thermafil the maximum tag penetration depth was, respectively, $109 \mu \mathrm{m}$ and $55 \mu \mathrm{m}$. Furthermore, the shape of the tags for GG and TG was different; rectangular for GC and circular for TG.

\section{Chemical Analysis Findings}

A spectrum taken from a tag at WL-5 and WL-2 for the two groups was likened at a spectrum of Thermafil $(\boldsymbol{-}$ Fig. 5) and GuttaCore gutta-percha ( - Fig. 6) and at a spectrum of Ah plus sealer showing their chemical analysis. This allowed us to affirm that the TG and GG tags consist of gutta-percha.

\section{Discussion}

To perform tridimensional filling, the essential requirements are a previous tridimensional shaping and tridimensional cleaning. Obviously, this task is much more difficult in the case of oval-shaped root canals. To fill suitably an ovalshaped root canal, the void and the sealer areas should be as small as possible, and this is possible at the condition that gutta-percha is thermoplastic.

The present study was designed to quantify the GPFA, SFA, and VA on the teeth filled using two different techniques of carrier-based obturation, Thermafil and GuttaCore, with the goal to provide a measure of quality. The Thermafil obturator consists of two parts: the plastic carrier, derivative of polysulfone; and the gutta-percha, whereas GuttaCore consists of a carrier manufactured from a cross-linked, thermoset elastomer of gutta-percha coated in gutta-percha. The presence of the plastic carrier in a Thermafil obturator could make a retreatment and/or the postspace preparation a difficult task. ${ }^{25,26}$ What's more, the clinicians who lack the basic principles for use Thermafil obturator could perform a root canal filling with a naked plastic carrier in the last millimeter of the canal.

Indeed, the GuttaCore carrier was designed to facilitate removal during retreatment and/or postspace preparation by fast, simply, and safe trephining through the core. ${ }^{27-29}$

Moreover, from a clinical point of view, our internal data (preclinical hands-on on different filling techniques, Strasbourg University) showed that the learning curve to master the GuttaCore filling is faster than the learning curve to master the Thermafil technique, with less possibility of

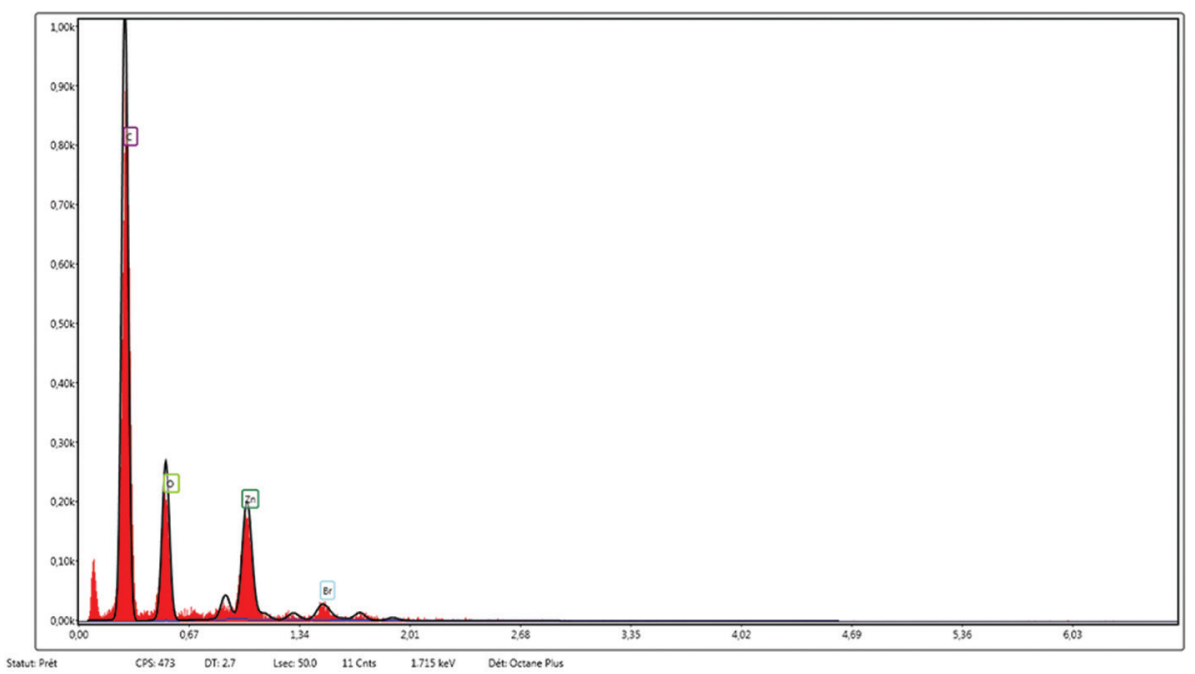

Fig. 5 Energy-dispersive X-ray spectrum of the Thermafil material. Major peak can be seen indicating $\mathrm{C}$ and smaller peaks indicating $\mathrm{O}$, Zn, and $\mathrm{Br}$. 


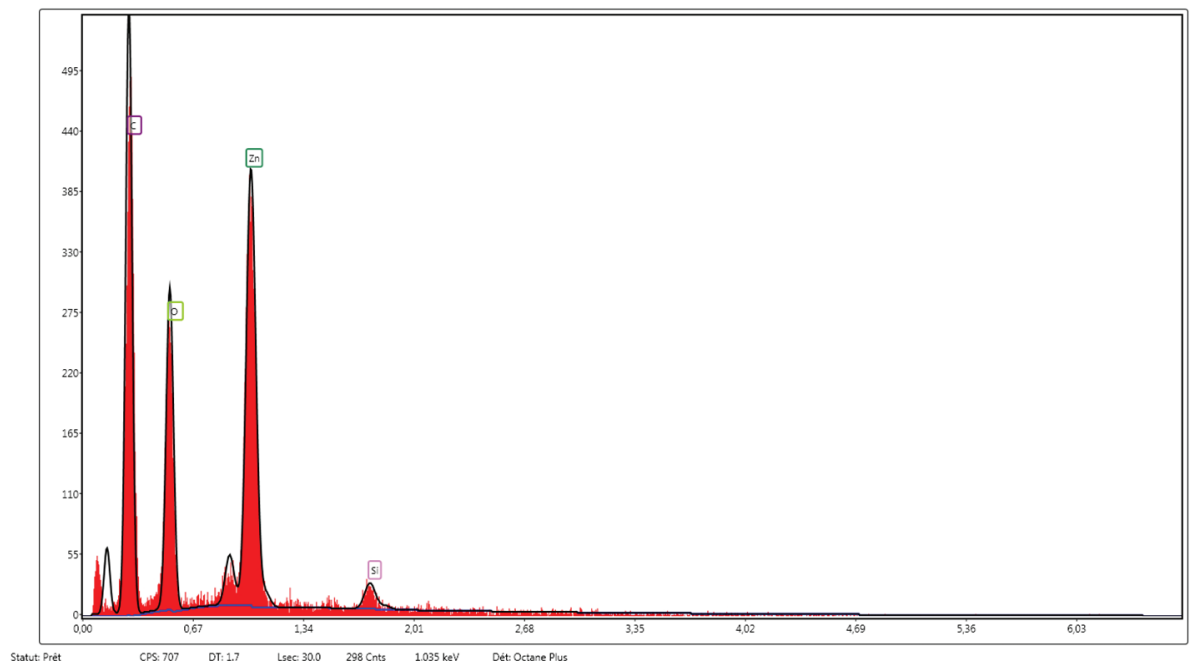

Fig. 6 Energy-dispersive X-ray spectrum of the GuttaCore material. Major peaks can be seen indicating C, Zn and O, and smaller peak indicating Si.

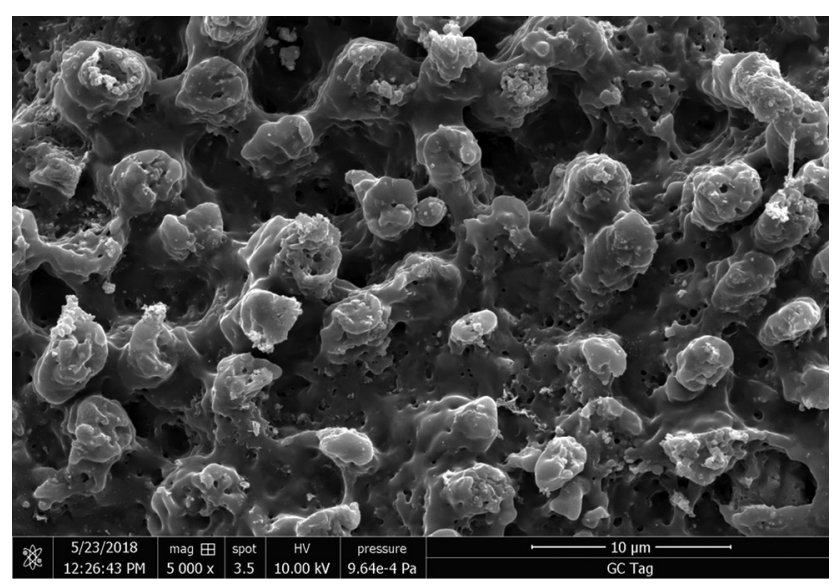

Fig. 7 Scanning electron micrograph showing the external surface of GuttaCore with the imprint of the dentinal tubules after previous decalcification and removal of dental tissues.

extrusion. In other words, the GuttaCore technique is less technique sensitive and less difficult to master compared with the Thermafil technique.

To reduce the possible errors, we decided to analyze each specimen using different instruments. At first, we choose the premolars by using radiographic and CBCT control images to demonstrate the good sampling homogeneity.

Afterward, specimens were observed using an optical numeric microscope at a magnification of $\times 500$. They were also observed by using SEM at magnifications up to $\times 30,000$, using direct observations and not replicates. Finally, we used a last control by using EDX analyses to know exactly the composition of the different filled areas, including tags.

At 5 and $2 \mathrm{~mm}$ distance from the apex, each specimen of both groups showed plasticized gutta-percha. No statistical difference was found for the GPFA, VA, and SAF values corresponding to the two tested thermo-plasticizing techniques.

Consequently, the null hypothesis, that there are no differences, at 5 and $2 \mathrm{~mm}$ from the apex, in the percentages of GPAF, VA, and SAF in oval-shaped root canals, filled by the Thermafil obturator and GuttaCore obturator using AH plus sealer was accepted. SEM micrographs, at 2 and $5 \mathrm{~mm}$ distances from the apex, disclosed the presence of tags for the GG, filling dentinal tubules. Moreover, SEM observations revealed the presence of tags for both groups. Gutta-percha tags ( - Fig. 7) could be able to block physically potentially remaining bacteria, so entombing them.

Chemical microanalyses allowed us to conclude that the tags of both groups were composed of gutta-percha material.

Of course, the present findings depend on the employed gutta-percha product qualities. Thermafil and GuttaCore gutta-percha is breakable at the solid state, but it changes on gluey, swollen proprieties when heated. The flowability of GC gutta-percha is higher than that of the standard gutta-percha of gutta-percha cones. ${ }^{30}$

At 2 and $5 \mathrm{~mm}$ distances from the apex, the present results reveal no statistically different filling ability for the two tested carrier-based techniques.

Moreover, at 2 and $5 \mathrm{~mm}$ distances from the apex, for each specimens treated using the two different filling techniques, the presence of gutta-percha tags was visualized.

Our results clearly show that the GuttaCore obturation can fill oval-shaped canals in an excellent way. Hence, we can state that GuttaCore system would be a filling technique less sensitive and less difficult to master compared with the Thermafil technique, preserving the same filling ability, in view of the fact that there was no difference, in terms of GPFA, SFA, and VA between the two different carrier-based obturation techniques.

\section{Funding}

None.

\section{Conflict of Interest}

None declared.

\section{References}

1 Krasner P, Rankow HJ. Anatomy of the pulp-chamber floor. J Endod 2004;30(1):5-16

2 Boveda C, Kishen A. Contracted endodontic cavities: the foundation for less invasive alternatives in the management of apical periodontitis. Endod Topics 2015;33:169-186 
3 Eaton JA, Clement DJ, Lloyd A, Marchesan MA. Micro-computed tomographic evaluation of the influence of root canal system landmarks on access outline forms and canal curvatures in mandibular molars. J Endod 2015;41(11):1888-1891

4 Alovisi M, Pasqualini D, Musso E, et al. Influence of contracted endodontic access on root canal geometry: an. in vitro. study. J Endod 2018;44(4):614-620

5 Mancino D, Kharouf N. Root canal treatment of dilacerated second maxillary premolars: planning the shaping procedure. J Clin Exp Dent 2018;10(6):e624-e627

6 Zehnder M. Root canal irrigants. J Endod 2006;32(5):389-398

7 Gu LS, Kim JR, Ling J, Choi KK, Pashley DH, Tay FR. Review of contemporary irrigant agitation techniques and devices. J Endod 2009;35(6):791-804

8 Kanisavaran ZM. Chlorhexidine gluconate in endodontics: an update review. Int Dent J 2008;58(5):247-257

9 Mohammadi Z, Abbott PV. The properties and applications of chlorhexidine in endodontics. Int Endod J 2009;42(4):288-302

10 Wu M, van der Sluis LW, Wesselink PR. A preliminary study of the percentage of gutta-percha-filled area in the apical canal filled with vertically compacted warm gutta-percha. Int Endod J 2002;35(6):527-535

11 De-Deus G, Gurgel-Filho ED, Magalhães KM, Coutinho-Filho T. A laboratory analysis of gutta-percha-filled area obtained using Thermafil, System B and lateral condensation. Int Endod J 2006;39(5):378-383

12 De-Deus G, Maniglia-Ferreira CM, Gurgel-Filho ED, Paciornik S, Machado AC, Coutinho-Filho T. Comparison of the percentage of gutta-percha-filled area obtained by Thermafil and System B. Aust Endod J 2007;33(2):55-61

13 Ørstavik D. Materials used for root canal obturation: Technical, biological and clinical testing. Endod Topics 2005;12:25-38

14 Peng L, Ye L, Tan H, Zhou X. Outcome of root canal obturation by warm gutta-percha versus cold lateral condensation: a meta-analysis. J Endod 2007;33(2):106-109

15 Whitworth J. Methods of filling root canals: principles and practices. Endod Topics 2005;12:2-24

16 Carver K, Nusstein J, Reader A, Beck M. In vivo antibacterial efficacy of ultrasound after hand and rotary instrumentation in human mandibular molars. J Endod 2007;33(9):1038-1043
17 Bukiet F, Couderc G, Camps J, et al. Wetting properties and critical micellar concentration of benzalkonium chloride mixed in sodium hypochlorite. J Endod 2012;38(11):1525-1529

18 Park E, Shen YA, Haapasalo M. Irrigation of the apical root canal. Endod Topics 2012;27:54-73

19 Wang Z, Shen Y, Haapasalo M. Effectiveness of endodontic disinfecting solutions against young and old Enterococcus faecalis biofilms in dentin canals. J Endod 2012;38(10):1376-1379

20 Ordinola-Zapata R, Bramante CM, Cavenago B, et al. Antimicrobial effect of endodontic solutions used as final irrigants on a dentine biofilm model. Int Endod J 2012;45(2):162-168

21 Haapasalo M, Endal U, Zandi H, Coil JM. Eradication of endodontic infection by instrumentation and irrigation solutions. Endod Topics 2005;10:77-102

22 Haapasalo M, Shen YA. Current therapeutic options for endodontic biofilms. Endod Topics 2012;22:79-98

23 Haapasalo M, Qian W, Shen YA. Irrigation: beyond the smear layer. Endod Topics 2012;27:35-53

24 Sundqvist G, Figdor D, Endodontic Treatment of Apical Periodontitis. In: Ørstavik D, Pitt Ford TR, eds. Essential Endodontology: Prevention and Treatment of Apical Periodontitis. Oxford: Blackwell; 1998

25 Wilcox LR. Thermafil retreatment with and without chloroform solvent. J Endod 1993;19(11):563-566

26 Wong AW, Zhang S, Li SK, Zhang C, Chu CH. Clinical studies on core-carrier obturation: a systematic review and meta-analysis. BMC Oral Health 2017;17(1):167

27 Beasley RT, Williamson AE, Justman BC, Qian F. Time required to remove guttacore, thermafil plus, and thermoplasticized gutta-percha from moderately curved root canals with protaper files. J Endod 2013;39(1):125-128

28 Rödig T, Wagner J, Wiegand A, Rizk M. Efficacy of the ProTaper retreatment system in removing Thermafil, GuttaCore or vertically compacted gutta-percha from curved root canals assessed by micro-CT. Int Endod J 2018;51(7):808-815

29 Schroeder AA, Ford NL, Coil JM. Micro-computed tomography analysis of post space preparation in root canals filled with carrier-based thermoplasticized gutta-percha. Int Endod J 2017;50(3):293-302

30 Greco K, Carmignani E, Cantatore G. The Thermafil root canal obturation system. G Ital Endod 2011;25:97-109 